\title{
The influence of thermodynamic phase on the retrieval of mixed-phase cloud microphysical and optical properties in the visible and near infrared region
}

\author{
Joonsuk Lee ${ }^{\mathrm{a}}$, Ping Yang ${ }^{\mathrm{a}}$, Andrew E. Dessler ${ }^{\mathrm{a}}$, \\ Bryan A. Baum ${ }^{\mathrm{b}}$ and Steven Platnick ${ }^{\mathrm{c}}$
}

a. Department of Atmospheric Sciences, Texas A\&M University, College Station, TX77843

b. NASA Langley Research Center, Science Directorate, Hampton, VA 23681

c. NASA Goddard Space Flight Center, Code 613.2, Greenbelt, MD 20771

Revised Manuscript (GRSL-00181-2005)

for publication in

IEEE Geoscience and Remote Sensing Letters

Corresponding author address: Dr. Ping Yang, Dept. of Atmospheric Sciences, Texas A\&M University, College Station, TX 77843. Tel: 979-845-4923; email: pyang@ariel.met.tamu.edu 


\begin{abstract}
Cloud microphysical and optical properties are inferred from the bidirectional reflectances simulated for a single-layered cloud consisting of an external mixture of ice particles and liquid droplets. The reflectances are calculated with a rigorous discrete ordinates radiative transfer model and are functions of the cloud effective particle size, the cloud optical thickness, and the values of the ice fraction in the cloud (i.e., the ratio of ice water content to total water content). In the present light scattering and radiative transfer simulations, the ice fraction is assumed to be vertically homogeneous; the habit (shape) percentage as a function of ice particle size is consistent with that used for the Moderate Resolution Imaging Spectroradiometer (MODIS) operational (Collection 4 and earlier) cloud products; and the surface is assumed to be Lambertian with an albedo of 0.03. Furthermore, error analyses pertaining to the inference of the effective particle sizes and optical thicknesses of mixed-phase clouds are performed. Errors are calculated with respect to the assumption of a cloud containing solely liquid or ice phase particles. The analyses suggest that the effective particle size inferred for a mixed-phase cloud can be underestimated (or overestimated) if pure liquid phase (or pure ice phase) is assumed for the cloud, whereas the corresponding cloud optical thickness can be overestimated (or underestimated).
\end{abstract}




\section{Introduction}

Both ice and water phase particles may coexist in clouds when the temperature is between $-40^{\circ} \mathrm{C}$ and $0^{\circ} \mathrm{C}[1],[2]$. Such mixed-phase clouds are important for several reasons. First, the difference in saturation vapor pressure between ice and water means that these clouds evolve differently from single-phase clouds. Additionally, mixed-phase clouds alter the radiative flux differently from single-phase clouds, thereby necessitating different treatment of clouds in both numerical weather forecasting and general circulation models (e.g., [3]). Furthermore, mixed-phase clouds can create hazardous conditions for aviation due to decreased visibility and aircraft icing [4], [5].

Mixed-phase clouds are frequently observed in the atmosphere [1], [6]. Recent research on mixed-phase clouds has focused on the analysis of ground-based or aircraft measurements [1], [6], the microphysical characteristics in cloud processes [7], [8], and modeling simulations [3], [9]. However, current operational satellite-based retrievals of cloud microphysical and optical properties are based on the assumption of a cloud being composed entirely of either ice particles or water droplets [10]-[12]. This study investigates the cloud optical and microphysical properties for clouds containing a mixture of both ice and water particles and compares them with retrievals that incorrectly assume that the phase is solely ice or liquid water.

\section{Radiative Transfer Model}

The radiative transfer model used in this study discretizes the atmosphere into 100 layers. The cloud-free atmospheric optical thickness for each layer is computed using the MODTRAN radiative transfer model [13]. The bidirectional reflectance at the top of the 
atmosphere is computed using the discrete-ordinate radiative transfer model (DISORT) [14] with a precalculated clear-sky atmospheric gaseous optical thickness vertical profile from MODTRAN. For a cloud layer, the bulk single-scattering properties of ice particles and water droplets are available from a precalculated library. The single-scattering properties of ice particles, covering a range of sizes from 1 to $4000 \mu \mathrm{m}$, are computed with the approach reported in [15]. The mean (or bulk) scattering properties are calculated using 30 in situ size distributions measured for a variety of midlatitude and tropical cirrus cloud systems obtained during several field campaigns [16], [17].

In this study, the particle habit (or shape) percentage as a function of particle size for a given size distribution of ice crystals is consistent with that used in the MODIS (Moderate Resolution Imaging Spectroradiometer) operational (Collection 4 and earlier) cloud products [18]. Specifically, the habits of ice particles considered for a given size distribution includes bullet rosettes, hollow columns, aggregates, and hexagonal plates. For small ice particles ( $D_{\max }<70 \mu \mathrm{m}$, where $D_{\max }$ is maximum dimension), the ice habit mixture is composed of $50 \%$ bullet rosettes, $25 \%$ hexagonal plates, and $25 \%$ hollow columns. For large ice particles $\left(D_{\max }>70 \mu \mathrm{m}\right)$, the mixture is composed of $30 \%$ aggregates, $20 \%$ bullet rosettes, $20 \%$ hexagonal plates, and $20 \%$ hollow columns, following [19] and [12]. The single-scattering properties of water clouds are calculated based on the Lorenz-Mie scattering, assuming 50 different Gamma size distributions.

The single-scattering properties of mixed-phase clouds are calculated following [20]. Here, the mixed-phase clouds are assumed to be a uniform mixture of ice and water particles. The total water content $(T W C)$ is the sum of ice water content $(I W C)$ and liquid 
water content $(L W C)$, i.e., $T W C=I W C+L W C$. Following [20], the ice mass fraction $(\gamma)$, is defined as follows:

$$
\gamma=\frac{I W C}{T W C}
$$

The effective particle size $D_{e m}$ for a mixed-phase cloud is derived in [20] by:

$$
D_{e m}=\left[\left(\frac{\gamma}{D_{e i} \rho_{i}}+\frac{1-\gamma}{D_{e w} \rho_{w}}\right) \rho_{m}\right]^{-1},
$$

where $D_{e}$ and $\rho$ indicate the effective size and mass density, respectively; and the subscripts $i, w$, and $m$ in Eq. (2) denote ice, water, and mixed-phase clouds, respectively. The optical thickness of a mixed-phase cloud $\left(\tau_{m}\right)$ is given by

$$
\tau_{m}=\tau_{i}+\tau_{w}=\Delta z \int Q_{e i} A_{i} n_{i} d D+\Delta z \int Q_{e w} A_{w} n_{w} d D
$$

where $\Delta z$ is the geometric thickness of the cloud, $Q_{e}$ is the extinction efficiency, $A$ is the projected area of the particle, $n$ is the size distribution of particle number density, $D$ is the characteristic length of the particle. It can be shown that the optical thicknesses pertaining to the ice and water components of a mixed-phase cloud are given, respectively, by the following expressions:

$$
\begin{gathered}
\tau_{i}=\frac{\left\langle Q_{e i}\right\rangle D_{e m} \rho_{m} \gamma}{\left\langle Q_{e m}\right\rangle D_{e i} \rho_{i}} \tau_{m}, \\
\tau_{w}=\frac{\left\langle Q_{e w}\right\rangle D_{e m} \rho_{m}(1-\gamma)}{\left\langle Q_{e m}\right\rangle D_{e w} \rho_{w}} \tau_{m},
\end{gathered}
$$

The detailed mathematical expressions for the quantities involved in Eqs. (4) and (5) can be found in [20].

\section{Methodology}


We calculate the bidirectional reflectance at the top of the atmosphere using the radiative transfer model discussed in the previous section. The bidirectional reflectance is defined as

$$
R\left(\mu, \phi ; \mu_{0}, \phi_{0}\right)=\frac{\pi I(0 ; \mu, \phi)}{\mu_{0} F_{0}},
$$

where $I(0 ; \mu, \phi)$ is the reflected radiance at the top of atmosphere, $F_{0}$ is the incident solar flux at the top of the atmosphere, $\mu_{0}$ is cosine of the solar zenith angle, $\phi_{0}$ is the solar azimuthal angle, $\mu$ is cosine of the sensor zenith angle, and $\phi$ is the sensor azimuthal angle. In this study, we simulate the bidirectional reflectance at high latitudes where mixed-phase clouds are frequently observed and use $55.8^{\circ}, 7.0^{\circ}$, and $20.0^{\circ}$ for the solar zenith angle, the sensor zenith angle, and the difference between solar and sensor azimuthal angles (i.e., the relative azimuth angle), respectively. The corresponding scattering angle is approximately $130^{\circ}$.

A popular method pioneered by [21], [10], and [22] infers cloud microphysical and optical properties by using the bidirectional reflectance observations in two or more spectral bands that have varying amounts of cloud particle absorption, e.g., a nonabsorbing visible band and an absorbing near infrared band, or even two shortwave infrared bands [11]. The reflectance in a nonabsorbing band (e.g., 0.67, 0.87um) is largely dependent on cloud optical thickness, whereas the reflectance in an absorbing band (e.g., 1.6, 2.1, and $3.75 \mu \mathrm{m}$ ) is sensitive to the cloud particle size [23]. In this study, we use a nonabsorbing and absorbing band defined by the MODIS spectral bandpasses at 0.87 and $2.13 \mu \mathrm{m}$, respectively, which is a combination used for the primary MODIS operational cloud retrieval [18]. 
This method is most accurate over a dark ocean surface away from sunglint, where the reflection comes from the cloud layer with little influence from the underlying surface [12]. On a bright surface such as ice or snow where the surface reflectance is large, the signal from the cloud is mixed with the signal from surface. In this study, a surface albedo of 0.03 is used both at $0.87 \mu \mathrm{m}$ and $2.13 \mu \mathrm{m}$ [24], [12] which is a value for dark ocean surface. Sunglint is not a factor for the solar-viewing geometry defined in this study.

\section{Results}

Figure 1 shows the variation of the effective particle diameter (Fig. 1(a)) and optical thickness (Fig. 1(b)) for clouds composed solely of ice, water, and a range of ice mass fractions. The water cloud has an effective particle diameter, $D_{e w}$, of $15 \mu \mathrm{m}$ and an optical thickness of $\tau_{w}=10$. The ice cloud has an effective particle diameter, $D_{e i}$, of $50 \mu \mathrm{m}$ and an optical thickness of $\tau_{i}=10$. In Fig. 1(a) when the ice fraction $\gamma=0$, i.e., a pure liquid water cloud case, the effective particle diameter becomes that of the water cloud. When $\gamma=1$, i.e., a pure ice cloud case, the effective diameter becomes that of the ice cloud. For values of $\gamma$ between 0 and 1 , the effective particle size varies smoothly between the ice and water cloud value. When $\gamma=0.5$, for example, the ice water content equals the liquid water content, but the effective particle diameter ( $23.5 \mu \mathrm{m}$, see Eq. 2$)$ is less than the arithmetic mean of the ice and water cloud effective particle sizes $(32.5 \mu \mathrm{m})$. This occurs because there are larger numbers of smaller particles in the water cloud. 
Fig. 1(b) shows similar results as for Fig. 1(a), but for a cloud with total optical thickness, $\tau_{m}$, equal to 10 . Note that the magnitude of the derivative of the $\tau_{w}$ and $\tau_{i}$ components increases rapidly with $\gamma$.

Figure 2 shows the response of the bidirectional reflectance at 0.87 and $2.13 \mu \mathrm{m}$ to changes in $\gamma$. These results are superimposed on theoretical isolines of $\tau$ and $D_{e}$ for pure ice (Fig. 2(a)) and pure water (Fig. 2(b)) clouds. Each symbol (+) corresponds to the ice fraction, which changes from 0 to 1 in increments of 0.1 . The reflectances change gradually between values corresponding to a homogeneous water and ice cloud. The reflectance at $2.13 \mu \mathrm{m}$ for a water cloud $(\gamma=0)$ is larger than that for the ice cloud $(\gamma=1)$ because (a) ice is more absorbing than liquid water, and (b) ice cloud particles tend to be larger than for the water cloud and therefore have a smaller cloud reflectance. Note that the cloud reflectance tends to decrease with increasing particle size. Thus, as $\gamma$ increases, the effective particle size inferred from the liquid water theoretical curves increases. The optical thickness in Fig. $2 \mathrm{~b}$ also increases with $\gamma$ because the ice particles have a larger asymmetry parameter due to their larger size and nonspherical habits.

Figure 3 compares the differences between homogeneous (with respect to thermodynamic phase) ice and water clouds for effective particle diameters (Fig. 3(a)) and optical thicknesses (Fig. 3(b)) retrieved from the theoretical relationships of Fig. 2. In this example $\left(D_{e i}=50 \mu \mathrm{m}, D_{e w}=15 \mu \mathrm{m}\right)$, the effective particle diameter inferred with the assumption of a homogeneous water cloud is smaller than the actual particle size of the mixed-phase cloud. Conversely, the effective particle diameter obtained assuming an ice cloud is larger than that of the mixed-phase cloud. Figure 3(b) shows the differences in 
the inferred optical thickness. For this case $\left(\tau_{m}=10\right)$, the optical thickness obtained with the water (ice) cloud assumption is larger (smaller) than that of the mixed-phase cloud.

Figure 4 shows the variation of the bidirectional reflectance at 0.87 and $2.13 \mu \mathrm{m}$ with both $\gamma$ and optical thickness for mixed-phase clouds as well as for homogeneous ice and water clouds. The effective particle diameter of the ice and water clouds is fixed at $50 \mu \mathrm{m}$ and $15 \mu \mathrm{m}$, respectively. The optical thickness varies from 1 to 15 . In Fig. 4(a) when $\gamma=1$, the reflectances are those values corresponding to the optical thickness of $1,3,5,8$, and 15 for ice clouds, respectively. In Fig. 4(b) when $\gamma=0$, the reflectances are those values corresponding to the optical thickness of $1,3,5,8$, and 15 for water clouds, respectively. It is clear from this plot that substantial errors in inferred effective particle size and optical thickness parameters may be incurred if a mixed-phase cloud is treated as a single-phase ice or water cloud when using a bispectral retrieval algorithm.

\section{Summary}

Mixed-phase clouds are a frequent phenomenon in the atmosphere and are important for several reasons. In this study, we have investigated the accuracy of the retrieval of two properties, effective particle size and optical thickness, for a single layered cloud containing both ice and water particles using the bidirectional reflectance at two wavelengths. The mixed-phase cloud is modeled as an external mixture of ice and water particles rather than as two distinct layers, each of which has hydrometeors of a single phase. Bidirectional reflectances are computed using a discrete ordinates radiative transfer model over an ocean surface having an albedo of 0.3 . Based on these simulated reflectances for both single-phase and mixed-phase clouds, the effective particle diameter 
and optical thickness are inferred. The sensitivity of these inferred values to the ice fraction is investigated. It is shown that the assumption of a single thermodynamic phase can induce errors in the retrieved parameters when the cloud is composed of both ice and water, since the bidirectional reflectances are influenced by the ice fraction. Specifically, the effective particle size retrieved for a mixed-phase cloud from the visible and nearinfrared bands can be underestimated (or overestimated) if pure water (or ice) phase is assumed, whereas the corresponding optical thickness of the mixed-phase cloud can be overestimated (or underestimated). While we present results for only one solar-satelliteviewing geometry, the sensitivity to ice fraction is expected to hold for viewing conditions that are unaffected by sunglint. Finally, we note that the identification of mixed-phase clouds as well as their discrimination from well-separated multiphase cloud layers (such as thin cirrus overlying a lower-level water cloud) remains a very challenging task for passive satellite sensors.

\section{Acknowledgements}

This research is supported by a NASA research grant (NNG04GL24G) and by the National Science Foundation (NSF) CAREER Award research grant (ATM-0239605). 


\section{References}

[1] R. P. Fleishauer, V. E. Larson, and T. H. Vonder Haar, "Observed microphysical structure of midlevel, mixed phase clouds", J. Atmos. Sci., vol. 59, pp. 1779-1804, 2002.

[2] A. V. Korolev, G. A. Isaac, S. G. Cober, J. W. Strapp, and J. Hallett, "Microphysical characterization of mixed-phase clouds", Q.J.R. Meteorol. Soc., vol. 129, pp. 39$65,2003$.

[3] Z. Sun and K. P. Shine, "Studies of the radiative properties of ice and mixed phase clouds", Q. J. R. Meteorol. Soc., vol. 120, pp. 111-137, 1994.

[4] J. T. Riley, "Mixed-phase icing conditions: a review", Technical Report of Federal Aviation Administration, DOT/FAA/AR-98/76, Washington DC, http://www.tc.faa.gov/its/act141/reportpage.html, 1998.

[5] S. G. Cober, G. A. Isaac, and J. W. Strapp, "Characterizations of aircraft icing environments that include supercooled large drops", J. Appl. Meteor., vol. 40, pp. 1984-2002, 2001.

[6] P. Zuidema, B. Barker, Y. Han, J. Intrieri, J. Key, P. Lawson, S. Matrosov, M. Shupe, R. Stone, and T. Uttal, "An arctic springtime mixed-phase cloudy boundary layer observed during SHEBA”, J. Atmos. Sci., vol. 62, pp. 160-176, 2005.

[7] M. Z. Jacobson, "Development of mixed-phase clouds from multiple aerosol size distributions and the effect of the clouds on aerosol removal", J. Geophys. Res., vol. 108(D8), 4245, 10.1029/2002JD002961, 2003.

[8] A. V. Korolev and G. A. Isaac, "Phase transformation of mixed-phase clouds", Q. J. R. Meteorol. Soc., vol. 129, pp. 19-38, 2003. 
[9] D. Lubin, "Thermodynamic phase of maritime Antarctic clouds from FTIR and supplementary radiometric data", J. Geophys. Res., vol. 109(D04204), 10.1029/2003JD003979, 2004.

[10] T. Nakajima and M. D. King, "Determination of the optical thickness and effective particle radius of cloud from reflected solar radiation measurements, Part I: Theory", J. Atmos. Sci., vol. 47, pp. 1878-1893, 1990.

[11] S. Platnick, J. Y. Li, M. D. King, H. Gerber, and P. V. Hobbs, "A solar reflectance method for retrieving the optical thickness and droplet size of liquid water clouds over snow and ice surfaces", J. Geophys. Res., vol. 106, pp. 15185-15199, 2001.

[12] M. D. King, S. Platnick, P. Yang, G. T. Arnold, M. A. Gray, J. C. Riedi, S. A. Ackerman, and K. N. Liou, "Remote sensing of liquid water and ice cloud optical thickness and effective radius in the arctic: Application of airborne multispectral MAS data", J. Atmos. Oceanic Technol., vol. 21, pp. 857-875, 2004.

[13] A. Berk, L. S. Bernstein, and D. C. Robertson, "MODTRAN: A moderate solution model for LOWTRAN-7", Report GL-TR-89-0122, Geoph. Lab. Air Force System Command, Hanscom AFB, USA, 1989.

[14] K. Stamnes, S.-C. Tsay, W. Wiscombe, and K. Jayaweera, "A numerically stable algorithm for discrete-ordinate-method radiative transfer in multiple scattering and emitting layered media”, Appl. Opt., vol. 27, pp. 2502-2509, 1988.

[15] P. Yang, K. N. Liou, K. Wyser, and D. Mitchell, "Parameterization of the scattering and absorption properties of individual ice crystals", J. Geophys. Res., vol. 105, pp. 4699-4718, 2000. 
[16] Q. Fu, "An accurate parameterization of the solar radiative properties of cirrus clouds for climate models", J. Climate, vol. 9, pp. 2058-2082, 1996.

[17] D. L. Mitchell, A. Macke, and Y. Liu, "Modeling cirrus clouds. Part II: Treatment of radiative properties", J. Atmos. Sci., vol. 53, pp. 2967-2988, 1996.

[18] S. Platnick, M. D. King, S. A. Ackerman, W. P. Menzel, B. A. Baum, J. C. Riedi, and R. A. Frey, "The MODIS cloud products: Algorithms and examples from Terra", IEEE Trans. Geosci. Remote Sens., vol. 41, pp. 459-473, 2003.

[19] B. A. Baum, D. P. Kratz, P. Yang, S. C. Ou, Y. Hu, P. F. Soulen, and S-C. Tsay, "Remote sensing of cloud properties using MODIS airborne simulator imagery during SUCCESS: 1. Data and models", J. Geophys. Res., vol. 105, pp. 11767-11780, 2000.

[20] P. Yang, H. L. Wei, B. A. Baum, H-L. Huang, A. J. Heymsfield, Y. X. Hu, B-C. Gao, D. D. Turner, "The spectral signature of mixed-phase clouds composed of nonspherical ice crystals and spherical liquid droplets in the terrestrial window region", JQSRT, vol. 79, pp. 1171-1188, 2003.

[21] S. Twomey and T. Cocks, "Remote sensing of cloud parameters from spectral reflectance measurements in the near-infrared", Beitr Phys. Atmos., vol. 62, pp. 172179, 1989.

[22] F. Rawlins and J. S. Foot, "Remotely sensed measurements of stratocumulus properties during FIRE using the $\mathrm{C} 130$ aircraft multi-channel radiometer", J. Atmos. Sci., vol. 47, pp. 2488-2503, 1990.

[23] King, M. D., "Determination of the scaled optical thickness of clouds from reflected solar radiation measurements", J. Atmos. Sci., vol. 44, pp. 1734-1751, 1987. 
[24] K. D. Moore, K. J. Voss, and H. R. Gordon, "Spectral reflectance of Whitecaps: Instrumentation, calibration, and performance in coastal waters", J. Atmos. Oceanic Technol., 15, 496-509, 1998. 


\section{Figure legends}

Figure 1. The variation of (a) effective particle diameter and (b) optical thickness as a function of ice fraction $(\gamma)$ in a mixed-phase cloud. Subscripts $i, w$, and $m$ refer to an ice, liquid water, and mixed-phase cloud, respectively.

Figure 2. The variation of the bidirectional reflectance of mixed-phase cloud at 0.87 and $2.13 \mu \mathrm{m}$ with the ice fraction $(\gamma)$ superimposed on the theoretical $\tau$ - $D_{\mathrm{e}}$ curves for the (a) ice cloud and (b) water cloud. $\gamma$ varies from 0 to 1 with the $(+)$ symbols from top to bottom.

Figure 3. The comparison of the (a) effective diameter and (b) optical thickness retrieved assuming a homogeneous ice and water cloud as a function of ice mass fraction $(\gamma)$. The true mixed-phase cloud parameters $\left(\tau_{\mathrm{m}}, D_{\mathrm{em}}\right)$ are also shown.

Figure 4. Same as Fig. 2 except that the optical thickness of mixed-phase cloud varies from 1 to 15. 

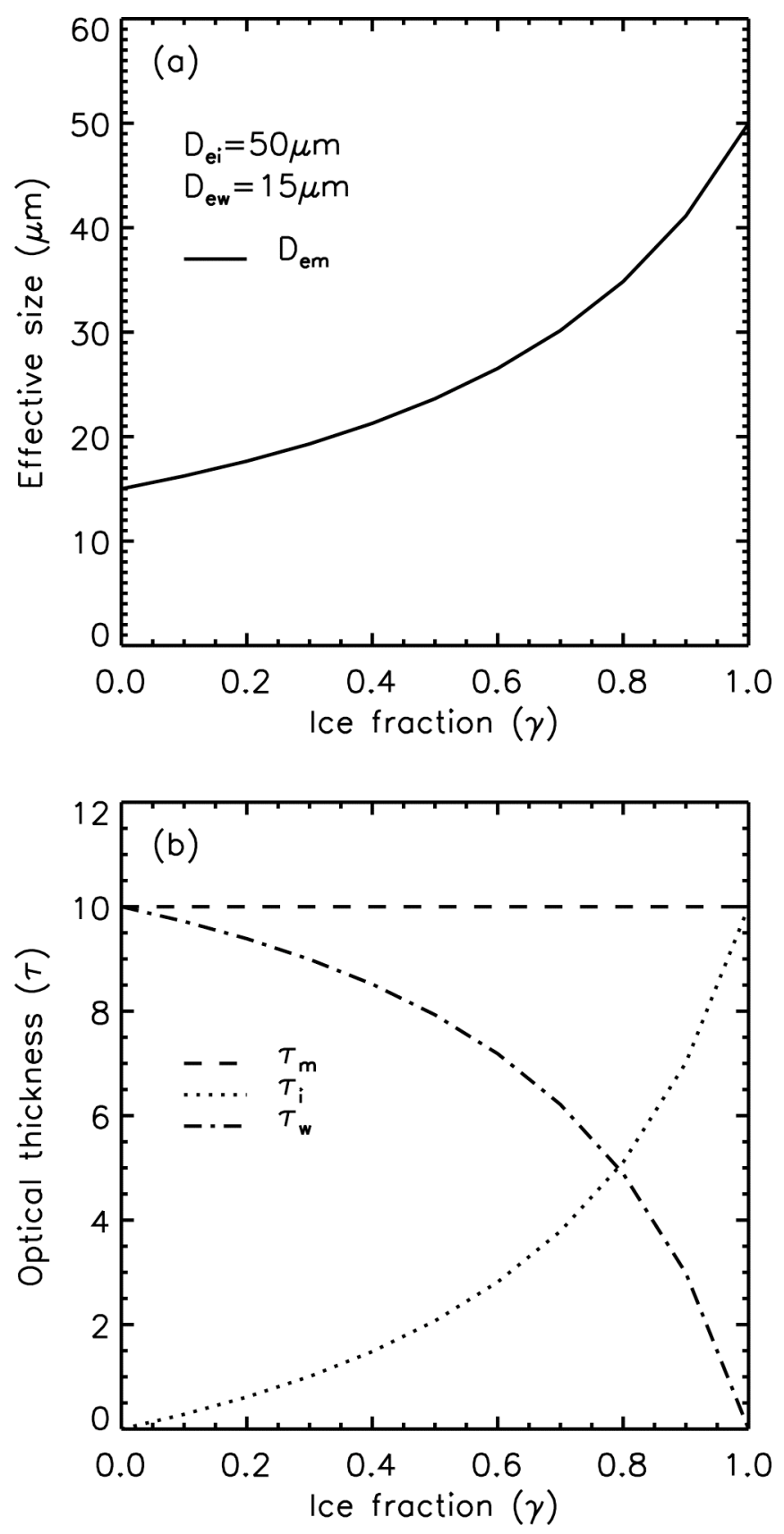

Figure 1. The variation of (a) effective particle diameter and (b) optical thickness as a function of ice fraction $(\gamma)$ in a mixed-phase cloud. Subscripts $i, w$, and $m$ refer to an ice, liquid water, and mixed-phase cloud, respectively. 

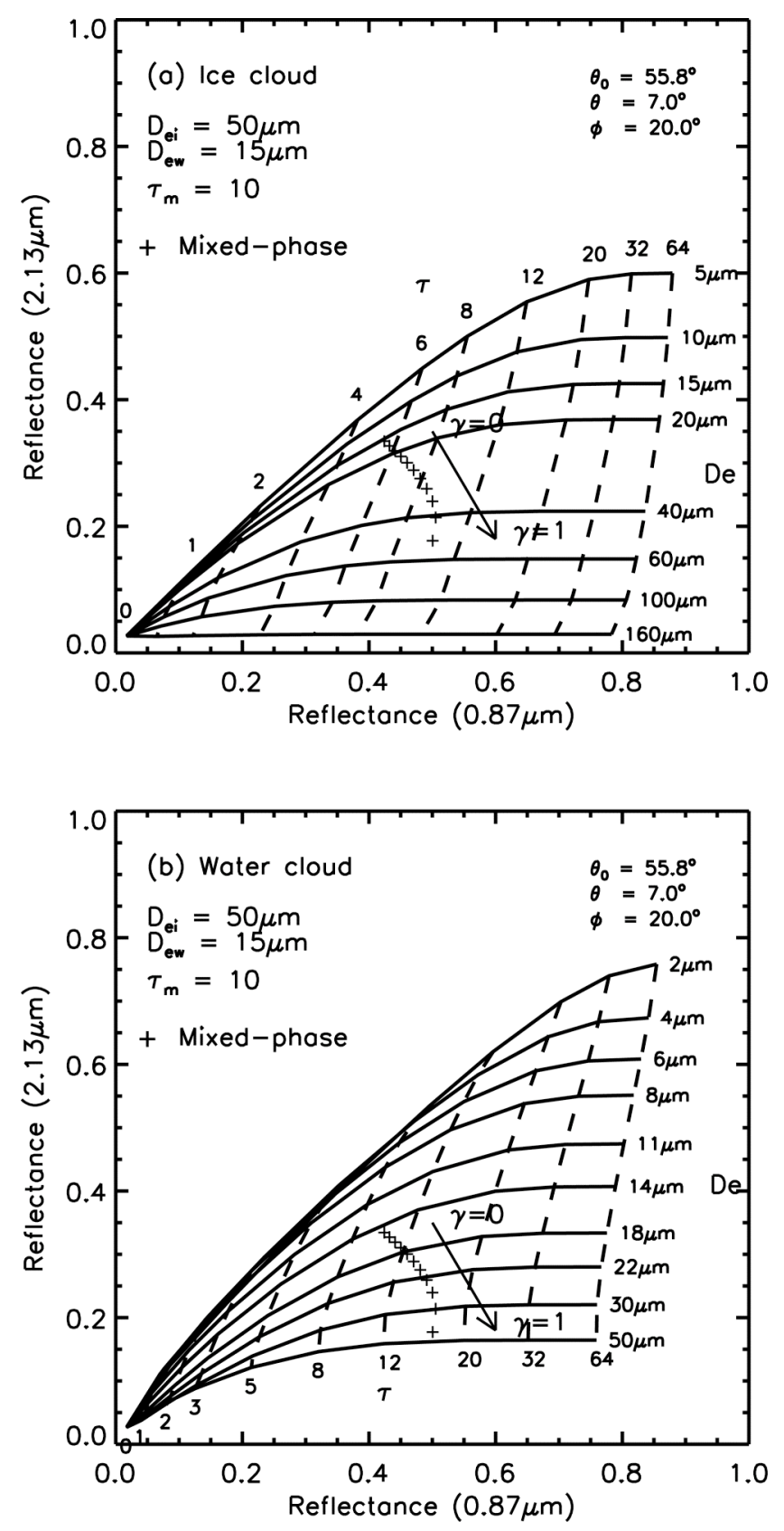

Figure 2. The variation of the bidirectional reflectance of mixed-phase cloud at 0.87 and $2.13 \mu \mathrm{m}$ with the ice fraction $(\gamma)$ superimposed on the theoretical $\tau$ - $D_{\mathrm{e}}$ curves for the (a) ice cloud and (b) water cloud. $\gamma$ varies from 0 to 1 with the $(+)$ symbols from top to bottom. 

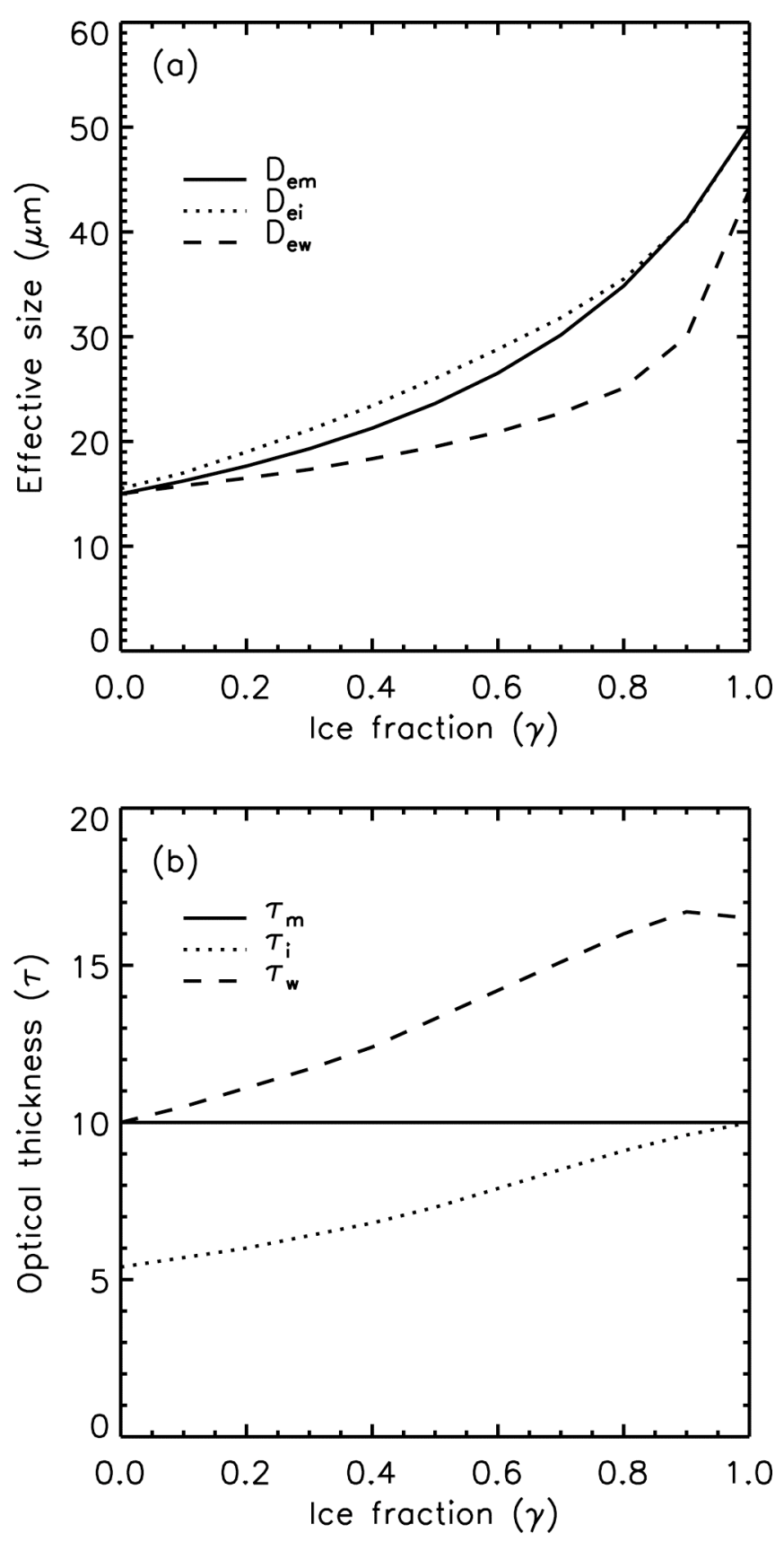

Figure 3. The comparison of the (a) effective diameter and (b) optical thickness retrieved assuming a homogeneous ice and water cloud as a function of ice mass fraction $(\gamma)$. The true mixed-phase cloud parameters $\left(\tau_{\mathrm{m}}, D_{\mathrm{em}}\right)$ are also shown. 

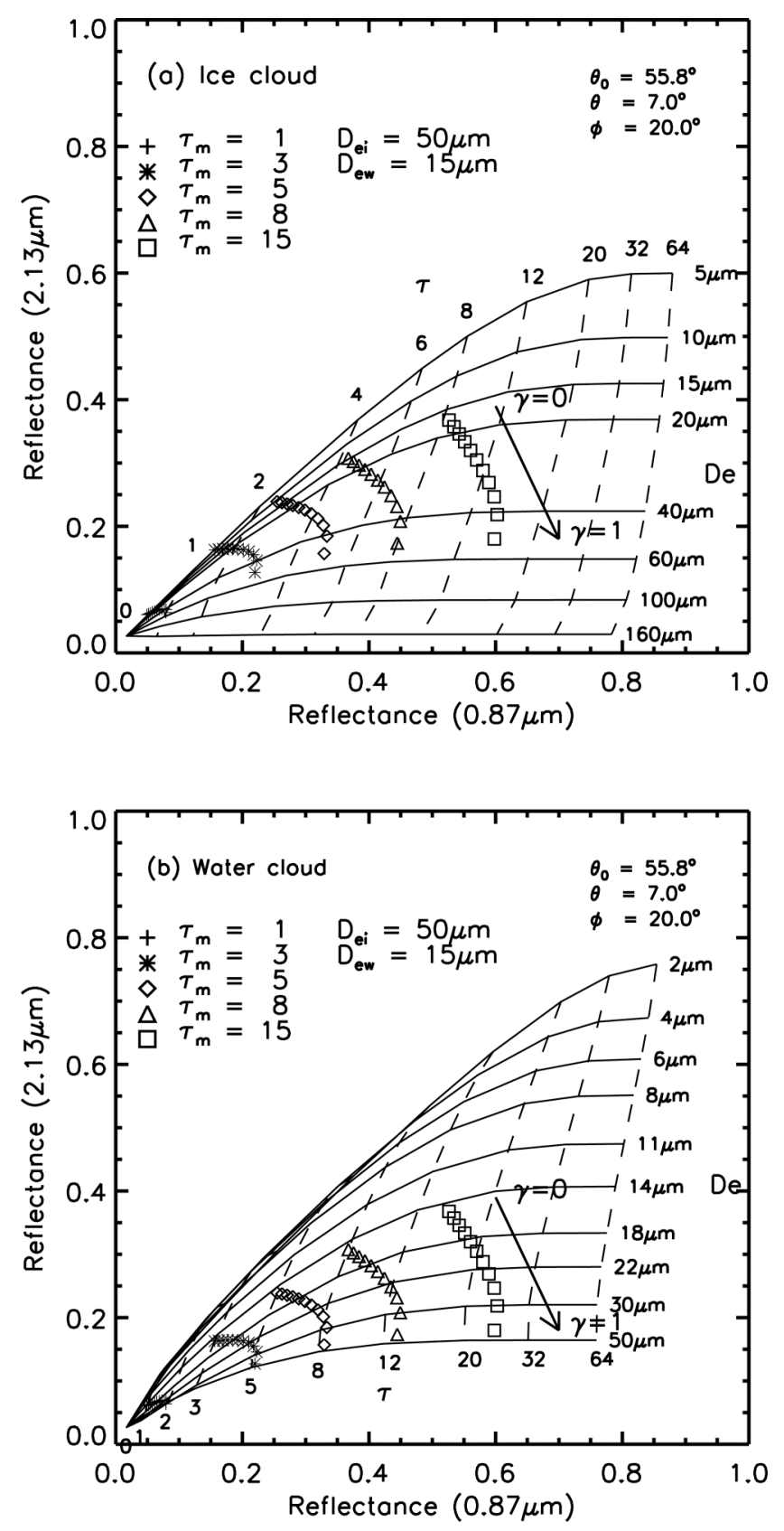

Figure 4. Same as Fig. 2 except that the optical thickness of mixed-phase cloud varies from 1 to 15 . 